\title{
Aquisição de dados de densidade e integração de dados de propriedades físicas da rocha em testemunhos de sondagem da Província Mineral de Carajás
}

Hiromi Nakashima, Lucas Martins Alves, Caio Francisco Pereira, Halana Pereira, Vitor Nascimento, Adalene Moreira Silva, Catarina L. Bemfica Toledo, Luiz Fernando Martins Ribeiro, Túlio Marques Soares, Luciano Mozer de Assis - Universidade de Brasília, Distrito Federal

\section{Copyright 2016, SBGf - Sociedade Brasileira de Geofísica}

Este texto foi preparado para a apresentação no VII Simpósio Brasileiro de Geofísica, Ouro Preto, 25 a 27 de outubro de 2016. Seu conteúdo foi revisado pelo Comitê Técnico do VII SimBGt, mas não necessariamente representa a opinião da SBGf ou de seus associados. É proibida a reprodução total ou parcial deste material para propósitos comerciais sem prévia autorização da SBGf.

\section{Resumo}

This projetct propose the development studies of iron formations based in physics feature of friables and plugs rocks from Carajás (PA).

To progress the objective has been taken the density measures of samples from N4WS in Província Mineral de Carajás, after that their were correlated with magnetic susceptibility and geochesmistry. Although has had two types of samples, friables and plugs, what demanded diferentes ways of measurements. For plugs samples were based in Arquimedes method with water and air at regular balance while friables used sabe method, but in a pentapycnomether. The last one needed a math correction to aproximated to in situ density.

The results were great to distinguish the litotypes by their physics feature and depth. Consequently, the results also confirm the possibility to use geophysics methods sinse the answear were continuing.

\section{Introdução}

O minério de ferro é a principal commodity brasileira no âmbito econômico, sendo o maior bem de exportação nacional. Dentre as reservas nacionais destaca-se a Província Mineral de Carajás (PMC), uma província polimetálica $(\mathrm{Mn}, \mathrm{Cu}, \mathrm{Au}, \mathrm{Ni}, \mathrm{U}, \mathrm{Ag}, \mathrm{Pd}, \mathrm{Pt}, \mathrm{Os}$, dentre outros), onde o minério de ferro de alto teor é basicamente composto por hematita (60-70\% Fe). O estudo de depósitos de minério de ferro tem avançado significativamente na última década no cenário mundial e a utilização de ferramentas como aerogeofísica de alta resolução, principalmente, a aerogravimetria gradiométrica possibilitou um incremento tecnológico, descoberta de novos alvos mineralizados e uma otimização da pesquisa em brownfield e near mine através da modelagem e inversão dos dados. (e.g. Braga, 2009, Zacchi, 2010, Assis, 2013; Uendro, 2014; Ferreira, 2014) No entanto, a aquisição de dados de propriedades física de rochas é chave para análise e interpretação dos resultados.

A Província Mineral de Carajás (PMC), onde está inserida a área de trabalho, localiza-se na região sudeste do estado do Pará, limitada a leste pelos rios AraguaiaTocantins, oeste pelo rio Xingu, norte pela serra do Bacajá e a sul pela Serra dos Gradaús (DOCEGEO 1988). Apresenta, de forma geral, idade arqueana, uma estruturação WNW-ESSE e foi dividida em dois blocos tectônicos distintos, denominados por Tassinari \& Macambira (2004) de: Bloco Carajás, também conhecido como Cinturão Itacaiúnas (Docegeo, 1988), e o Bloco Xingu-Iricoumé. Santos (2006) considera a PMC dividida no domínio de idade Mesoarqueana denominado de Terreno Granito-Greenstone de Rio Maria e no domínio Neoarqueano (2,76-2,55 Ga) de Carajás, sendo o último uma província independente, havendo consenso que a região de Carajás constitui o principal núcleo arqueano do Cráton Amazônico, representando a porção crustal mais antiga e melhor preservada do cráton. Para melhor clareza quanto ao entendimento do trabalho, adotou-se a proposta de Santos (2003). O Domínio Rio Maria é caracterizado por uma crosta juvenil mesoarqueana, com sequências de greenstone belts e granitóides tipo TTG. O Domínio Carajás é uma região de crosta continental predominantemente neoarqueana, caracterizada principalmente por sequências metavulcanosedimentares e granitóides de alto K. Nesse domínio, aflora o Grupo Grão Pará que e compreende rochas metavulcânicas máficas e félsicas associadas a metassedimentos e lentes de formação ferrífera bandada, incluindo jaspilitos com minério de ferro. A Formação Carajás, uma das unidades do Grupo Grão Pará, é constituída formações ferríferas bandadas de fácies óxido do tipo jaspilito, com variações decorrentes de alterações, quase totalmente transformada em minério hematítico até uma profundidade de algumas centenas de metros, e cortada por diques e soleiras de rochas máficas. É a unidade que hospeda os depósitos de minério de ferro a um teor médio de $66 \%$ de ferro.

Afim de aprimorar os estudos geofísicos de formações ferríferas, este trabalho compreende analisar e interpretar os dados de densidade do minério de ferro, protominério e encaixantes do depósito de ferro da Província Mineral de Carajás. Correlacionando com os dados de susceptibilidade magnética e geoquímica adquiridas em trabalhos anteriores (Ferreira, 2014) para encontrar um padrão no comportamento físico dessas rochas e proporcionar um possível uso de métodos geofísicos no campo de exploração e caracterização do minério em questão.

\section{Metodologia}

A análise da densidade foi realizada em onze testemunhos de sondagem do corpo mineralizado N4WS de 2 em 2 metros, totalizando aproximadamente 1800 amostras. Como os furos de sondagem seccionaram porções alteradas, semi-alteradas e frescas do Grupo Estes são divididos em dois tipos, sendo o primeiro os friáveis no início dos furos, com o predomínio de canga de minério, canga química, máficas decompostas e o 
minério (hematitito friável), e o segundo em profundidade (variável conforme o furo) com a presença de rochas compactas, que predominam os jaspilitos e rochas máficas indiferenciadas (Figura 3).

Em função das diferentes condições físicas das amostras, foi proposto dois métodos diferentes para adquirir os valores de densidade. $O$ primeiro foi feito a densidade das rochas compactas na balança ToledoIND560 (Figura 1) e desenhada para facilitar a aquisição em testemunhos de sondagem. A densidade foi obtida pelo com base no princípio de Arquimedes através de valores obtidos da massa seca da rocha e da massa com os poros preenchidos de água e calculados a partir da seguinte formulação:

$$
D a=\left(\frac{M s}{M s-M m}\right) D
$$

Da, Ms, Mm, D são a densidade da amostra, massa da amostra seca, massa da amostra submersa e densidade da água, respectivamente.

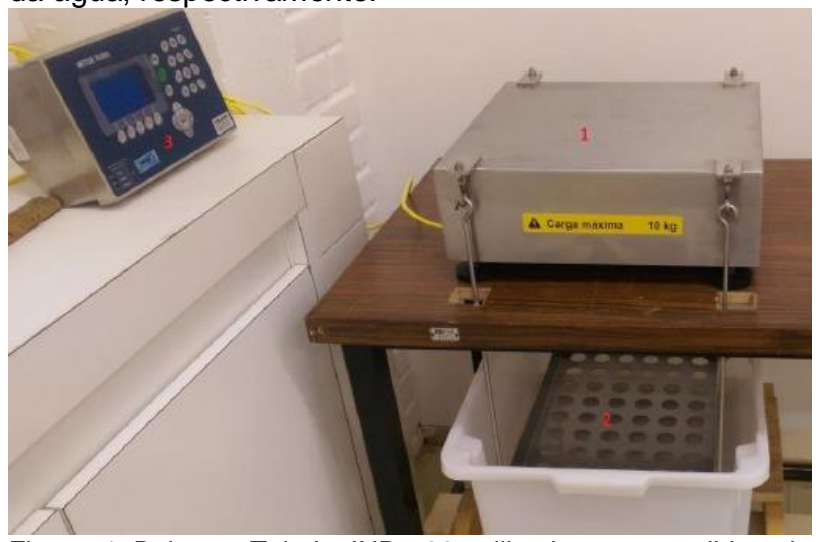

Figura 1 Balança Toledo IND-560 utilizada para medidas de densidade. 1- Base a seco; 2-Base imersa; 3-Painel de valores da amostra em questão.

Devido à necessidade de submergir a amostra, foi inviável usar o método anterior para as amostras em friáveis, desta maneira as amostras foram medidas no Pentapyc 5200e (Figura 2). Para a medição selecionavase, aproximadamente, 20 gramas de cada amostra (medidas na balança SHIMADZU AUW2200) que eram colocadas nas cápsulas e medidas dentro do equipamento durante aproximadamente 25 minutos. $O$ equipamento é designado para quantificar o volume verdadeiro de um material sólido por meio do princípio de Arquimedes de deslocamento de fluidos e de expansão de gás (Lei de Boyle). Como esse valor da densidade desconsidera os espaços vazios, ou seja, fornece a densidade média dos minerais que compõe a rocha, é necessário fazer uma análise comparativa da densidade, profundidade da amostra e geoquímica a fim de encontrar um comportamento padrão nos litotipos para posteriormente comparar com a densidade local, adquirida por meio de conhecido como perfilagem de densidade gama-gama. Os raios gama são utilizados pela perfilagem geofísica, devido a sua capacidade de penetração em material denso. O perfil registra as variações, em contagens por segundo, das densidades das camadas com a profundidade, pois existe uma relação entre a contribuição volumétrica de cada elemento constituinte e a densidade total da rocha (Kearey, 2002). O perfil geofísico gama-gama avalia a densidade a partir da leitura de valores de contagens por segundo, posteriormente convertidas para densidade, com taxas de aquisição variáveis. É fundamental um tratamento estatístico dos dados para a determinação de um valor único de densidade para cada unidade estudada (Almeida, 2011; Fonseca, 2014).

Os dados foram processados utilizando a extensão Target do software Oasis Montaj versão 8.5.

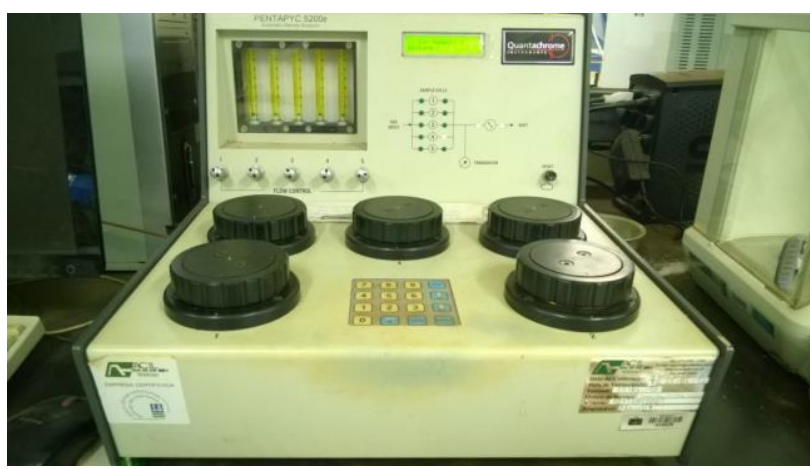

Figura 2 Pentapicnômetro Pentapyc 5200e. Observar os cinco compartimentos para inserção de amostras friáveis para coleta de dados de densidade. A proposta é a medição da densidade de partículas.

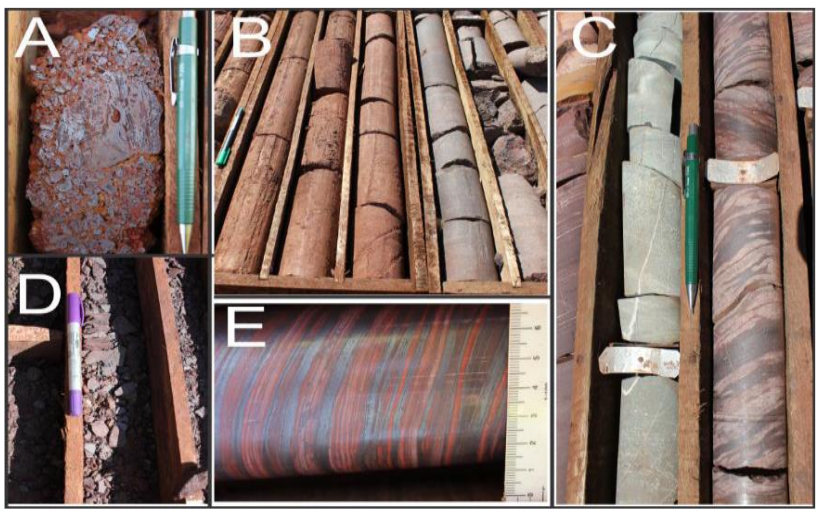

Figura 3 Principais litotipos da área de estudo. A) Canga de Minério; B) Canga Química variando para máfica semidecomposta; C) À esquerda rocha máfica e à direita o jaspilito; D) Detalhe do minério de ferro, denominado de hematita friável; E) Detalhe do Jaspilito com bandas de jaspe e óxido de ferro (Ferreira, 2014). 


\section{Resultados}

Congruente aos métodos, os resultados obtidos foram de dois tipos, os resultados por meio da balança e os por meio do pentapicnômetro.

As amostras compactas apresentam os resultados nas figuras $4 a$ e $4 b$, nela observa as propriedades físicas e a porcentagem de ferro total presente nos furos $1100 \mathrm{e}$ 1495.

Observa-se que os jaspilitos correspondem a altos teores de ferro, consequentemente possui uma elevada densidade e susceptibilidade magnética. No fim do furo 1495 (figura 4b) há um comportamento anômalo com os jaspilitos onde o teor de ferro total e a densidade se mantém elevada, mas a susceptibilidade cai drasticamente, isso ocorre pois inicialmente os jaspilitos são ricos em magnetita ( $\mathrm{Fe} 3 \mathrm{O} 4)$ que com o decorrer do tempo sofrem oxidação e transforma-se em hematita ( $\mathrm{Fe} 2 \mathrm{O} 3)$.

Junto dos jaspilitos, nota-se o comportamento para as rochas máficas, as quais em função da sua composição química possui baixo teor de ferro total e susceptibilidade magnética, consequentemente apresenta baixo valor de densidade.

Diferente das rochas compactas, os friáveis possuem uma elevada variação de litotipos. Na tabela 1 são apresentados os valores da média de densidade adquirida pelo pentapicnômetro, podendo observar inicialmente que as amostras com os valores de densidade mais elevada são o minério seguido dos jaspilitos, porém as cangas também se destacam. Além disso, observa que a máfica semidecomposta sofre os maiores valores de desvio padrão, podendo compreender a provável ação do intemperismo que alterou a composição original das amostras.

Ao comparar os valores de densidade medidos com outros trabalhos (tabela 2), observou uma diferença elevada dos valores, tornando-os ineficiente para uso de campo, pois os valores encontrados são distintos dos in situ, de forma que é necessária a uma correção para aproximar os valores encontrados com os valores in situ. (a)

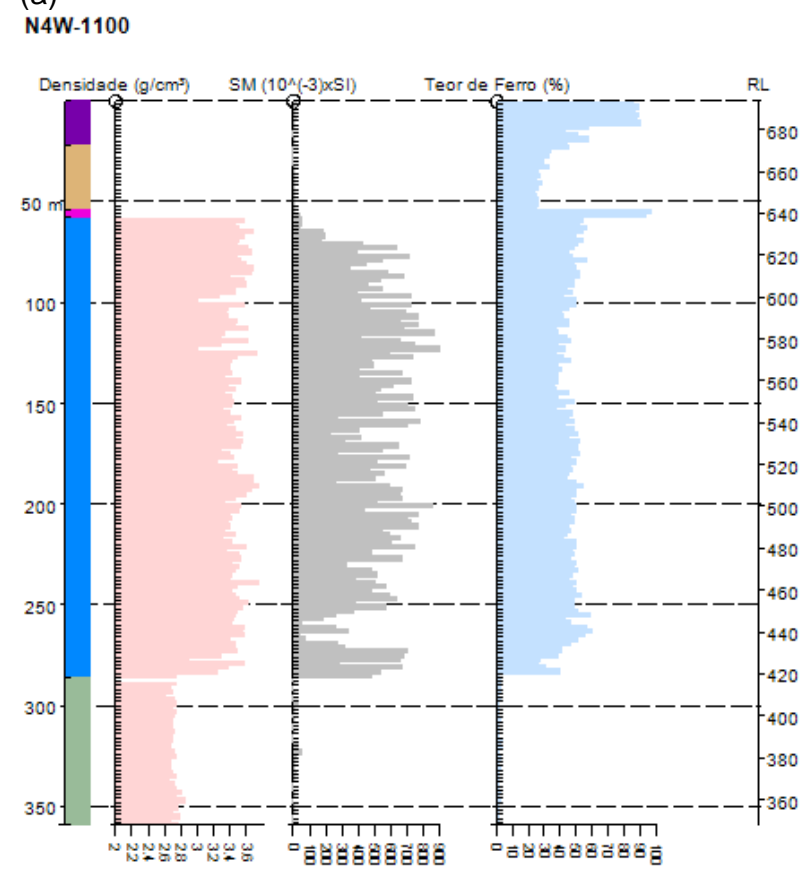

(b)

\section{N4WS-1495}

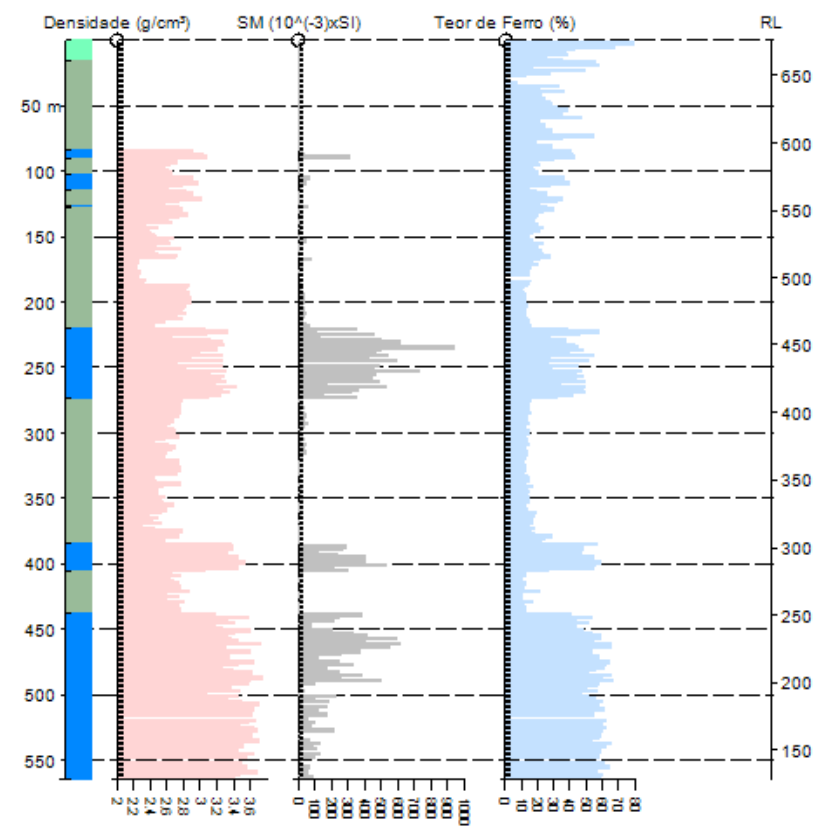

Figura 4- Gráficos que correlacionam unidades mapeadas com medidas de densidade $(\mathrm{g} / \mathrm{cm} 3)$, susceptibilidade magnética e teor de ferro(\%) dos furos de sondagem (a)N4WS-1100 e (b) N4WS-1495 
Tabela 1- Média de densidade conforme os litotipos mapeados através dos diferentes furos de sondagem estudados dentro do projeto.

\begin{tabular}{|c|c|c|c|}
\hline Furo & Litologia & Média & Desvio \\
\hline \multirow[t]{5}{*}{ N4W-F1012 } & $\mathrm{CQ}$ & 3,77 & 0,26 \\
\hline & $\mathrm{HF}$ & 4,95 & 0,22 \\
\hline & $\mathrm{JP}$ & 5,07 & 0,40 \\
\hline & MS & 3,29 & 0,42 \\
\hline & Geral & 3,50 & 0,65 \\
\hline \multirow[t]{3}{*}{ N4W-F1023 } & $\mathrm{CM}$ & 5,04 & 0,41 \\
\hline & $\mathrm{HF}$ & 5,21 & 0,17 \\
\hline & Geral & 5,18 & 0,23 \\
\hline \multirow[t]{4}{*}{ N4W-F1051 } & $\mathrm{CM}$ & 4,72 & 0,50 \\
\hline & $\mathrm{HF}$ & 5,46 & 0,30 \\
\hline & $J P$ & 4,85 & 0,00 \\
\hline & Geral & 5,39 & 0,39 \\
\hline \multirow[t]{5}{*}{ N4W-F1065 } & $\mathrm{CM}$ & 5,23 & 0,29 \\
\hline & $C Q$ & 3,70 & 0,07 \\
\hline & $\mathrm{HF}$ & 5,00 & 0,20 \\
\hline & MS & 3,66 & 0,61 \\
\hline & Geral & 4,61 & 0,84 \\
\hline \multirow[t]{4}{*}{ N4W-F1100 } & $\mathrm{CM}$ & 4,59 & 0,76 \\
\hline & $J P$ & 5,57 & 0,05 \\
\hline & MS & 3,35 & 0,09 \\
\hline & Geral & 3,97 & 0,87 \\
\hline \multirow[t]{4}{*}{ N4W-F1115 } & CQ & 4,80 & 0,58 \\
\hline & $J P$ & 4,71 & 0,46 \\
\hline & MS & 3,50 & 0,32 \\
\hline & Geral & 4,17 & 0,74 \\
\hline \multirow[t]{2}{*}{ N4W-F1279 } & MS & 3,14 & 0,22 \\
\hline & Geral & 3,14 & 0,22 \\
\hline \multirow[t]{4}{*}{ N4W-F1348 } & $\mathrm{CM}$ & 4,38 & 0,00 \\
\hline & $\mathrm{CQ}$ & 3,31 & 0,06 \\
\hline & MS & 3,13 & 0,17 \\
\hline & Geral & 3,25 & 0,28 \\
\hline \multirow[t]{4}{*}{ N4W-F1398 } & $\mathrm{CM}$ & 3,88 & 0,20 \\
\hline & $C Q$ & 3,39 & 0,10 \\
\hline & $\mathrm{HF}$ & 5,29 & 0,00 \\
\hline & Geral & 3,68 & 0,59 \\
\hline \multirow[t]{3}{*}{ N4W-F1495 } & $\mathrm{CQ}$ & 3,48 & 0,04 \\
\hline & MS & 3,02 & 0,25 \\
\hline & Geral & 3,07 & 0,27 \\
\hline \multirow[t]{6}{*}{ N4W-F1515 } & $\mathrm{CM}$ & 4,69 & 0,29 \\
\hline & $C Q$ & 3,64 & 0,22 \\
\hline & $\mathrm{HF}$ & 5,10 & 0,25 \\
\hline & $J P$ & 3,60 & 0,34 \\
\hline & MS & 3,48 & 0,56 \\
\hline & Geral & 3,91 & 0,74 \\
\hline
\end{tabular}

Tabela 2- Média dos valores de densidade utilizados por Assis ( 2013) para modelagem de dados aerogravimétricos gradiométricos.

\begin{tabular}{|c|l|c|}
\hline Sigla & \multicolumn{1}{|c|}{ Litotipo } & $\begin{array}{c}\text { Densidade média } \\
\left(\mathrm{g} / \mathrm{cm}^{3}\right)\end{array}$ \\
\hline HC & Hematitito compacto & 3,61 \\
\hline HF & Hematitito friável & 3,12 \\
\hline HMN & Hematitito manganesifero & 3,13 \\
\hline FMN & Formação ferrifera manganesifera & 3,17 \\
\hline JP & Jaspilito & 3,18 \\
\hline SO & Solo & 2,03 \\
\hline CM & Colúvio / elúvio de hematitito lateritizado & 3,09 \\
\hline CQ & Lateritas & 2,58 \\
\hline MD & Saprolito das rochas máficas, horizonte A/B & 1,95 \\
\hline MSD & Saprolito das rochas máficas, horizonte C/R & 2,34 \\
\hline MS & Rochas máficas indiferenciadas & 2,58 \\
\hline QZ & Veio de Quartzo & 2,47 \\
\hline AET & Arenito & 2,62 \\
\hline CGL & Conglomerado & 2,93 \\
\hline
\end{tabular}

Os dados da figura 5 representa a relação da densidade dos friáveis dos furos de sondagem N4WS-1012, N4WS1100 e N4WS-1495 como exemplo.

No furo 1012, figura 5a, destaca-se em função de ser composto basicamente de friáveis. Ao analisar a densidade média geral $\left(3,5 \mathrm{~g} / \mathrm{cm}^{3}\right)$ apresentada na tabela 2 e comparar com a densidade dos outros furos, observase que é um dos menores valores. Isso ocorre função ao predomínio de máficas semidecompostas, porém os picos de densidade no início ( 0 a $60 \mathrm{~m}$ ) e entre 270 a 280 metros de profundidade deve-se a presença de canga química, hematitito friável e jaspilitos.

Ao analisar a figura 5b observa altos valores de densidade, pois há o predomínio de canga de minério (no início até 20 metros de profundidade) e jaspilito (aproximadamente 60 metros de profundidade, onde termina as amostras friáveis) enquanto no centro apresenta valores mais baixos em função das máficas semidecompostas. O pico dos valores de densidade do jaspilito são congruentes ao esperado, pois se comparar com os dados apresentado na figura 3a apresenta o mesmo pico no início das amostras compactas.

No furo 1495 (figura $5 c$ ) há somente dois tipos de friáveis, a canga química e a máfica semidecomposta, intercalado por amostras compactas. A densidade se mantêm mais padronizada, porém baixa ao se comparar com as amostras compactas (figura 3b), o que provavelmente é em função ao alto intemperismo. 


\section{N4W-1012}

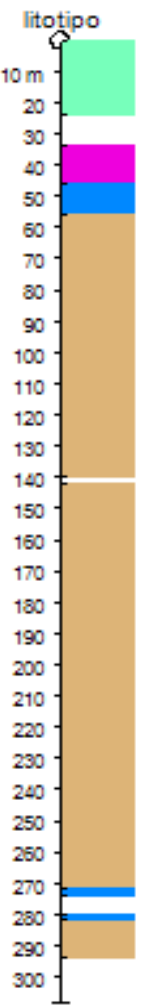

(a)

\section{N4W-1100}

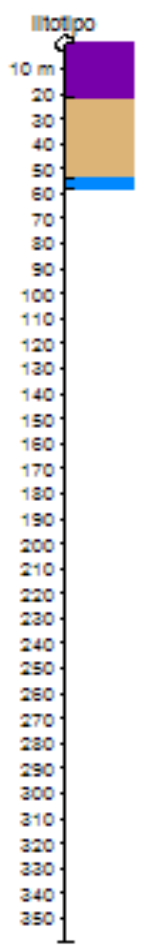

(b)

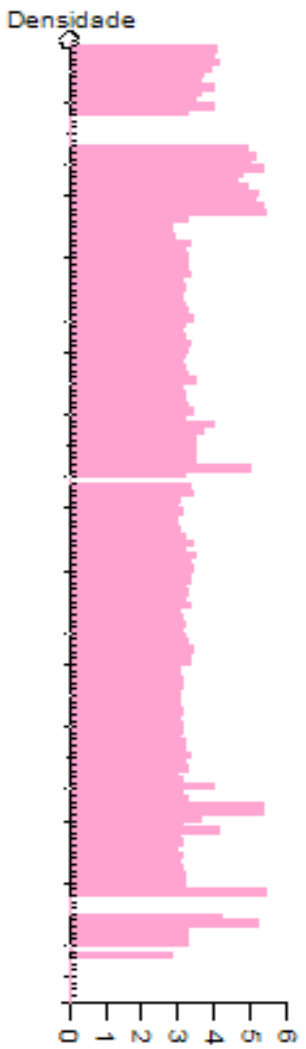

(c)

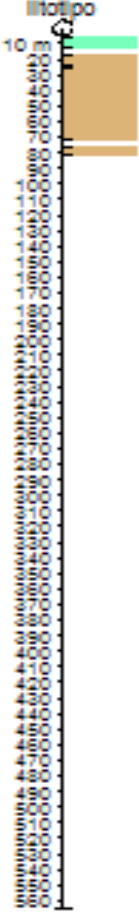

Figura 5 - Os gráficos mostram a correlação entre a parte friável dos furos de sondagem N4WS-1100, N4WS-1012, estudados e os dados de densidade obtidos pelo pentapicnometro. (A) Relação de friáveis do furo 1012; (B) Relação de friáveis do furo 1100 e densidade; (c) relação de friáveis do furo 1495 e densidade.

\section{Discussão e Conclusões}

Os resultados obtidos pelos dois métodos de coleta de densidade foram satisfatórios para diferenciar os litotipos presentes no corpo mineralizado N4W, além de apresentar uma diferenciação devido à profundidade, consequentemente à compactação do material. $\mathrm{E}$ necessário realizar a correção dos dados do método do pentapicnômetro para uma melhor correlação dos materiais friáveis com os compactos na extensão dos testemunhos de sondagem. Além de possibilitar a utilização dos dados para delimitação do corpo através de inversão geofísica, já que os dados precisam conter o mesmo padrão de informação. Apesar desses dados de densidade necessitar de correções, pois não consideram a porosidade e a estruturação, o pentapicnômetro é um excelente método para questão mineralógica, já que este considera o mineral que compõe a amostra. 


\section{Agradecimentos}

Os autores agradecem a empresa Vale através do Projeto de Cooperação Técnico-Cientifica entre a Universidade de Brasília e a Vale, bem como os Laboratórios de Geofísica Aplicada e de Propriedades Físicas do IG-UnB, bem como o Laboratório de Solos da Faculdade de Tecnologia. Professora A.M.Silva agradece ao CNPq pela bolsa de produtividade em pesquisa e os discentes de mestrado agradecem ao CNPq e CAPES pelas bolsas de estudo. Agradecemos ao ESRI-ArcGis pelas licenças acadêmicas dentro do Projeto de Cooperação com o IG-UnB e a Geosoft-Latino America pelo apoio constante.

\section{Referências}

ASSIS, Luciano M. et al. Geração de Modelo Exploratório para o Minério de Ferro da Província Mineral de Carajás Através da Integração de Dados Multifone (Dissetação de Mestrado em Geologia) - UNB

BEISIEGEL, V.R., Bernardelli, A.L., Drummond, N.F., Ruff, A.W., Tremaine, J.W. 1973. Geologia e recursos minerais da Serra dos Carajás: Revista Brasileira de Geociências, v. 3: 215-242.

COELHO, C.E.S. 1986. Depósito de ferro da Serra dos Carajás, Pará. Principais Depósitos Minerais Brasileiros Ferro e Metais da Indústria do Aço. DNPM, v. 2: 29-64. DOCEGEO. 1988. Revisão litoestratigráfica da Província Mineral de Carajás: Proceedings of Congresso Brasileiro de Geologia 35th, Sociedade Brasileira de Geologia: Belém, Brazil, p. 11-54.

FERREIRA, Vitor Nascimento. Caracterização de formações ferríferas bandadas através de dados de propriedades físicas de rocha e sua integração com dados aerogeofísicos: o estudo de caso do corpo N4WS, Serra Norte - Província Mineral de Carajás. 2014. 92 f., il. Dissertação (Mestrado em Geologia)-Universidade de Brasília, Brasília, 2014.

FIGUEIREDO E SILVA, Cristina et al. Evolução do Minério de Ferro Hidrotermal nos depósitos da Serra Norte Província Mineral Carajás (Tese de Doutorado) UFMG

KEAREYL P., BROOKSL M., HILL I. Geofísica de exploração, (Editora oficina de textos)

LOBATO, L.M., ROSIÈRE, C.A., Silva, R.C.F., Zucchetti. M., Baars, F.J., Seoane, J.C.S., Rios, F.J., Pimentel, M., Mendes, G.E., Monteiro, A.M. 2005a. A mineralização hidrotermal de ferro da Província Mineral de Carajás Controle estrutural e contexto na evolução metalogenética da província, in Marini, O. J., Queiroz, E. T., Ramos, B. W., Caracterização de depósitos minerais em distritos mineiros da Amazônia: DNPM/CTMineral/FINEP/ADIMB, Brasília, p. 25-92.
MACAMBIRA J.B. 2003. O ambiente deposicional da Formação Carajás e uma proposta de modelo evolutivo para a Bacia Grão Pará. Tese de Doutorado, Instituto de Geociências, Universidade Estadual de Campinas, 217p.

RESENDE N.P., e Barbosa A.L.M. 1972. Relatório de pesquisa de minério de ferro, distrito ferrífero da Serra dos Carajás, estado do Pará. AMZA, relatório final de pesquisa, v. 1, texto, 248 p, v. 2, mapas e seções geológicas, p. 119.

ZUCCHETTI, Marcia et al. Rochas máficas do grupo Grão Pará e sua relação com a mineralização de ferro dos depósitos N4 e N5, Carajás, PA (Tese de Doutorado) - UFMG 